\title{
Hemophagocytic Lymphohistiocytosis after Initiation of Combined Immunotherapy for Metastatic Melanoma
}

\author{
Gambichler T*, Abu Rached N, Nowack N, Behle \\ $B$ and Susok L \\ Department of Dermatology, Ruhr-University Bochum, \\ Germany \\ *Corresponding author: Thilo Gambichler, \\ Department of Dermatology, Ruhr-University Bochum, \\ Gudrunstrasse 56, 44791 Bochum, Germany
}

Received: February 11, 2021; Accepted: March 22, 2021; Published: March 29, 2021

\begin{abstract}
Hemophagocytic Lymphohistiocytosis (HLH), which is a severe, potentially fatal condition characterized by $\mathrm{T}$ lymphocyte overactivation, is predominantly caused by infections, hematological malignancies, and autoimmune conditions. $\mathrm{HLH}$ due to therapy with Immune Checkpoint Inhibitors (ICI) has rarely been reported. We describe a 60-year-old male with metastatic melanoma who developed HLH after the initiation of nivolumab plus ipilimumab treatment. Prompt diagnosis and high-dose mono-prednisolone therapy resulted in rapid resolution of his subjective symptoms and laboratory findings. Apart from this case presentation we provide a brief overview on clinical characteristics of previously observed $\mathrm{ICl}$-induced $\mathrm{HLH}$ cases. Given the increasing use of $\mathrm{ICl}$ in a variety of cancers, the frequency of HLH will very likely raise. HLH morbidity and mortality are often the result of delayed diagnosis and inappropriate treatment. Hence, $\mathrm{HLH}$ must be considered in $\mathrm{ICl}$-treated cancer patients who present with symptoms such as fever, cytopenias and hyperferritinemia.
\end{abstract}

Keywords: Hemophagocytic lymphohistiocytosis; Metastatic melanoma; Immune checkpoint inhibitors

\section{Introduction}

The therapy paradigms and outcomes of patients with cancer have dramatically changed since the introduction of immunotherapy with Immune Checkpoint Inhibitors (ICI), including cytotoxic T lymphocyte antigen-4 (CTLA-4) and programmed cell death protein (PD-1) and its ligand (PD-L1) antibodies. However, these agents can trigger immune-related Adverse Events (irAEs), which may be due to hyperactivation of $\mathrm{T}$ lymphocytes against self-antigens. Unlike the frequently described organ systems affected by irAEs, such as skin, gut, lungs, and endocrine system, hematologic toxicities have been poorly reported, probably due to their uncommon nature and lack of correct detection. One of these rare hematologic complications of ICI represents Hemophagocytic Lymphohistiocytosis (HLH), which is a severe, potentially fatal condition characterized by $\mathrm{T}$ lymphocyte overactivation that is measurable, for example, by soluble Interleukin-2 Receptor (IL-2R) determination in blood [1,2]. Primary or hereditary HLH (e.g., A91V mutation in PRF1) must be differentiated from secondary $\mathrm{HLH}$, which is predominantly caused by infections, malignancies, and autoimmune conditions, frequently in the context of underlying immunodeficiency or immunosuppression. HLH is usually characterized by recurrent high fever, splenomegaly, pancytopenia, hyperferritinemia, hypertriglyceridemia, hypofibrinogenemia, increased IL-2R, and hemophagocytosis on bone marrow assessment [1-10]. Here we report a patient with metastatic melanoma who developed HLH after initiation of anti-CTLA-4/PD-1 treatment and give a brief tabular overview on previously reported cases.

\section{Case Presentation}

We report a 60-year-old man with stage III BRAF-wildtype melanoma, who had received 8 cycles nivolumab $(240 \mathrm{mg}$ fix dose every other week) in the adjuvant setting. Since he showed disease progress on Computed Tomography (CT), including left axillary, pre-pectoral, and mediastinal lymph node metastases, combined immunotherapy with nivolumab (1mg/kg three weekly) and ipilimumab $(3 \mathrm{mg} /$ $\mathrm{kg}$ three weekly) was initiated. Nine weeks (three cycles) after the introduction of combi-immunotherapy, the patient was admitted to our skin cancer center with a history of high-spiking fever $\left(>38.5^{\circ}\right)$ and malaise. Blood cultures, procalcitonin, C-reactive protein, and virus serology for herpes simplex, herpes zoster, cytomegaly, HIV, and Epstein-Barr did not reveal evidence for an infection. PCR for SARS-CoV was negative on several test times. Remarkable laboratory findings included: Leukocytes $3670 / \mu \mathrm{l}(4600-9500)$, erythrocytes 3.8 mill/ $\mu \mathrm{l}$ (4.6-6.2), thrombocytes $139.000 / \mu \mathrm{l}(150.000-400.000)$, ferritin $12.806 \mathrm{ng} / \mathrm{ml}$ (30-400), fibrinogen $166 \mathrm{mg} / \mathrm{dl}$ (200-400), IL-2R 5999 $\mathrm{U} / 1$ (<710), lactate dehydrogenase $346 \mathrm{U} / \mathrm{l}$ (135-225), and up to 3-fold elevated liver transaminases. Antinuclear autoantibodies (ANA, dsDNA, ENA) were within the normal range. A CT-scan showed splenomegaly $(14.8 \mathrm{~cm})$ and regredient lymph node metastases. Bone marrow biopsy did not show signs of phagocytosis. Hence, the patient met 6 of 8 diagnostic criteria of HLH according to the HLH-2004 guideline. His Hscore was 189 (almost 80\% HLH probability). ICI was discontinued, and prednisolone was introduced in a tapered dose regimen (initial dosage $250 \mathrm{mg}$ ), resulting in a rapid decrease of the patient's clinical complaints and improvement of laboratory findings. After two weeks the patient has been fully recovered. His following outpatient treatment was performed with orally administered prednisolone tapered down to $20 \mathrm{mg} / \mathrm{d}$ for two weeks. Regular monitoring of his clinical performance and blood tests were carried out on a weekly basis.

\section{Discussion}

The present case clearly fulfilled the diagnostic criteria for 
Table 1: Characteristics of cancer patients $(n=69)$ with Immune Checkpoint Inhibitor (ICl) induced Hemophagocytotic Lymphohistiocytosis (HLH) [1-10, present case].

\begin{tabular}{|c|c|}
\hline Gender & \\
\hline Male & $\mathrm{n}=50 / 69(72.3 \%)$ \\
\hline Female & $\mathrm{n}=19 / 69(27.7 \%$ \\
\hline Median age (years) & $60(26-81)$ \\
\hline Cancer type & \\
\hline Melanoma & $\mathrm{n}=39(56.5 \%)$ \\
\hline Lung cancer & $\mathrm{n}=9(13 \%)$ \\
\hline Bladder cancer & $\mathrm{n}=4(5.8 \%)$ \\
\hline Renal cell cancer & $\mathrm{n}=4(5.8 \%)$ \\
\hline Miscellaneous & $\mathrm{n}=13(18.8 \%)$ \\
\hline ICI at HLH onset & \\
\hline Anti-CTLA-4 & $\mathrm{n}=7(10.1 \%)$ \\
\hline Anti-PD-1 & $\mathrm{n}=38(55.1 \%)$ \\
\hline Anti-PD-1/CTLA-4 & $\mathrm{n}=16(23.2 \%)$ \\
\hline Miscellaneous & $\mathrm{n}=8(11.6 \%)$ \\
\hline Median time (weeks) of HLH onset after ICl initiation & $6 \mathrm{weeks} \mathrm{(1-428)}$ \\
\hline HLH deaths & $11 / 68(16.2 \%)$ \\
\hline
\end{tabular}

secondary HLH $[1,2]$. Supported by the current literature [1-10], we are convinced that ICI was the cause of HLH in the present case. Of course, we cannot fully exclude that HLH was mainly driven by the underlying malignancy [11-16]. However, malignancy-triggered HLH occurs most frequently in T- and NK-cell lymphoma or leukemia, diffuse large B-cell lymphoma, and Hodgkin's disease [15]. Solid tumors are rarely associated with HLH with only a $3 \%$ prevalence in adults. Whereas causes of secondary HLH, including malignancies, infections, and autoimmune diseases, are due to an underlying condition, ICI-induced HLH has been recognized as a new clinical challenge in cancer management. Notably, HLH was also reported in patients managed with chimeric antigen receptor $\mathrm{T}$-cell therapy, further highlighting the association between T-cell activity modulation and HLH development [11-16].

Noseda et al. [1] and Dupré et al. [2] recently published data of ICIinduced HLH patients which extracted from large pharmacovigilance databases. Noseda et al. [1] searched the VigiBase including 49.883 ICI-related suspected adverse drug reactions and found 38 patients with HLH, whereas rates were highest in France $(0.4 \%)$ and the lowest in the USA (0.03\%). Dupré et al. [2] identified 5 ICI-induced HLH cases from a pharmacovigilance databases in the Parisian region. They published their 5 cases together with a review of the literature including 17 previously published patients [2]. Including the present case, we have studied new ICI-induced HLH cases recently published and collected the data with the aforementioned investigations as shown in Table $1[1,2]$. Remarkably, ICI-induced HLH is associated with a substantial male predominance, which was also observed in other clinical settings such as infection or malignancy-associated HLH [13,14]. In systematic review on ICI-induced irAEs [17], no differences in gender distribution have recently been found in hematologic irAEs. However, most cases were associated with irAEs such as hemolytic anemia and immune thrombocytopenic purpura [17]. Unsurprisingly, most patients with ICI-induced HLH had melanoma, for which ICI approvals exist much longer when compared to other cancer entities [1,2]. Table 1 also demonstrates that there is no difference between the immunotherapy agents (e.g., anti-PD-1, anti-CTLA-4) regarding the potency to cause HLH. Even though the onset of HLH may occur at any time under ICI treatment, it appears that early onset of HLH is more likely. Most importantly, however, fatal outcome was reported in $16.2 \%$ of ICI-induced HLH cases - a rate that seems to be smaller than the death rates (up to $50 \%$ ) reported for patients with HLH associated with other causes [1-10].

Basic scientists recently found a reciprocal correlation between PD-1 expression and tumor-associated phagocytic activity of macrophages as well as an enhanced cancer cell phagocytosis by macrophages following PD-1/PD-L1 targeting [9,11]. As also demonstrated in the present case, some patients with ICI-induced HLH improve with corticosteroids alone not requiring cytoreductive regimens such as etoposide [18]. Treatment approaches for more severe HLH cases include anti-interleukin 6 (e.g., tocilizumab) and anti-CD25 antibodies. Paradoxically, ICI (e.g., nivolumab) treatment has also successfully been used to treat relapsed/refractory EpsteinBarr virus-associated HLH [19]. Re-challenge of ICI after HLH resolution is a difficult clinical decision. In most cases previously reported, ICI was permanently discontinued. In patients with progressive cancer, however, ICI must be reconsidered if no treatment alternatives are available.

\section{Conclusion}

In conclusion, HLH morbidity and mortality are often due to delayed diagnosis and inappropriate treatment. Hence, HLH must be considered in ICI-treated cancer patients who present with symptoms such as fever, cytopenias, and hyperferritinemia.

\section{Acknowledgements}

The authors would like to thank the patient giving us informed consent for publishing his interesting case.

\section{Financial \& Competing Interest Disclosure}

TG has received speakers and/or advisory board honoraria from BMS, Sanofi-Genzyme, MSD, Novartis Pharma, Roche, Abbvie, Almirall, Janssen, Lilly, Pfizer, Pierre Fabre, Merck-Serono, outside the submitted work. LS has received speakers and/or advisory board honoraria from BMS, Sun-Pharma, MSD, and Novartis.

\section{Ethical Conduct of Research}

The authors state that they have followed the principles outlined in the Declaration of Helsinki for all human or animal experimental investigations. In addition, for investigations involving human subjects, informed consent has been obtained from the participant involved.

\section{Informed Consent Disclosure}

The authors state that they have obtained verbal and written informed consent from their patient for the inclusion of his medical and treatment history within this case report.

\section{Availability of Data and Materials}

All crucial data generated or analyzed during this case study are 
included in this published article.

\section{References}

1. Noseda R, Bertoli R, Muller L, Ceschi A. Haemophagocytic lymphohistiocytosis in patients treated with immune checkpoint inhibitors: analysis of WHO global database of individual case safety reports. J Immunother Cancer. 2019; 7: 117.

2. Dupré A, Michot JM, Schoeffler A, Frumholtz L, Baroudjian B, Delyon J, et al. Haemophagocytic lymphohistiocytosis associated with immune checkpoint inhibitors: a descriptive case study and literature review. $\mathrm{Br} \mathrm{J}$ Haematol. 2020; 189: 985-992.

3. Mizuta H, Nakano E, Takahashi A, Koyama T, Namikawa K, Yamazaki N Hemophagocytic lymphohistiocytosis with advanced malignant melanoma accompanied by ipilimumab and nivolumab: A case report and literature review. Dermatol Ther. 2020; 33: e13321.

4. Chin CK, Hall S, Green C, Van Hazel G, Spagnolo D, Cheah CY. Secondary haemophagocytic lymphohistiocytosis due to checkpoint inhibitor therapy. Eur J Cancer. 2019; 115: 84-87.

5. Honjo O, Kubo T, Sugaya F, Nishizaka T, Kato K, Hirohashi Y, Takahash $\mathrm{H}$, Torigoe $\mathrm{T}$. Severe cytokine release syndrome resulting in purpura fulminans despite successful response to nivolumab therapy in a patient with pleomorphic carcinoma of the lung: a case report. J Immunother Cancer. 2019; 7: 97.

6. Lorenz G, Schul L, Bachmann Q, Angermann S, Slotta-Huspenina J, Heemann $U$, et al. Hemophagocytic Iymphohistiocytosis secondary to pembrolizumab treatment with insufficient response to high-dose steroids. Rheumatology. 2019; 58: 1106-1109.

7. Thummalapalli R, Heumann T, Stein J, Khan S, Priemer DS, Duffield AS et al. Hemophagocytic Lymphohistiocytosis Secondary to PD-1 and IDO Inhibition in a Patient with Refractory Glioblastoma. Case Rep Oncol. 2020; 13: $508-514$.

8. Takahashi H, Koiwa T, Fujita A, Suzuki T, Tagashira A, Iwasaki Y. A case of pembrolizumab-induced hemophagocytic lymphohistiocytosis successfully treated with pulse glucocorticoid therapy. Respir Med Case Rep. 2020; 30: 101097.

9. Hantel A, Gabster B, Cheng JX, Golomb H, Gajewski TF. Severe hemophagocytic lymphohistiocytosis in a melanoma patient treated with ipilimumab + nivolumab. J Immunother Cancer. 2018; 6: 73 .

10. Özdemir BC, Latifyan S, Perreau M, Fenwick C, Alberio L, Waeber G, et al. Cytokine-directed therapy with tocilizumab for immune checkpoint inhibitorrelated hemophagocytic lymphohistiocytosis. Ann Oncol. 2020; 31: 17751778.

11. Kalmuk J, Puchalla J, Feng G, Giri A, Kaczmar J. Pembrolizumab-induced Hemophagocytic Lymphohistiocytosis: an immunotherapeutic challenge. Cancers Head Neck. 2020; 5: 3.

12. Davis EJ, Salem JE, Young A, Green JR, Ferrell PB, Ancell KK, et al. Hematologic Complications of Immune Checkpoint Inhibitors. Oncologist. 2019; 24: 584-588.

13. Yildiz H, Van Den Neste E, Defour JP, Danse E, Yombi JC. Adult haemophagocytic lymphohistiocytosis: a Review. QJM. 2020: hcaa011.

14. Ménard F, Besson C, Rincé $P$, Lambotte $O$, Lazure $T$, Canioni $D$, et al. Hodgkin lymphoma-associated hemophagocytic syndrome: a disorder strongly correlated with Epstein-Barr virus. Clin Infect Dis. 2008; 47: 531-534.

15. Daver N, McClain K, Allen CE, Parikh SA, Otrock Z, Rojas-Hernandez C, et al. A consensus review on malignancy-associated hemophagocytic lymphohistiocytosis in adults. Cancer. 2017; 123: 3229-3240.

16. Yeung SJ, Qdaisat A, Chaftari P, Lipe D, Merlin J, Rajha E, et al. Diagnosis and management of immune-related adverse effects of immune checkpoint therapy in the emergency department. J Am Coll Emerg Physicians Open. 2020; 1: 1637-1659.

17. Triggianese P, Novelli L, Galdiero MR, Chimenti MS, Conigliaro P, Perricone $\mathrm{R}$, et al. Immune checkpoint inhibitors-induced autoimmunity: The impact of gender. Autoimmun Rev. 2020; 19: 102590.

18. Takahashi H, Koiwa T, Fujita A, Suzuki T, Tagashira A, Iwasaki Y. A case of pembrolizumab-induced hemophagocytic lymphohistiocytosis successfully treated with pulse glucocorticoid therapy. Respir Med Case Rep. 2020; 30: 101097

19. Liu P, Pan X, Chen C, Niu T, Shuai X, Wang J, et al. Nivolumab treatment of relapsed/refractory Epstein-Barr virus-associated hemophagocytic lymphohistiocytosis in adults. Blood. 2020; 135: 826-833. 